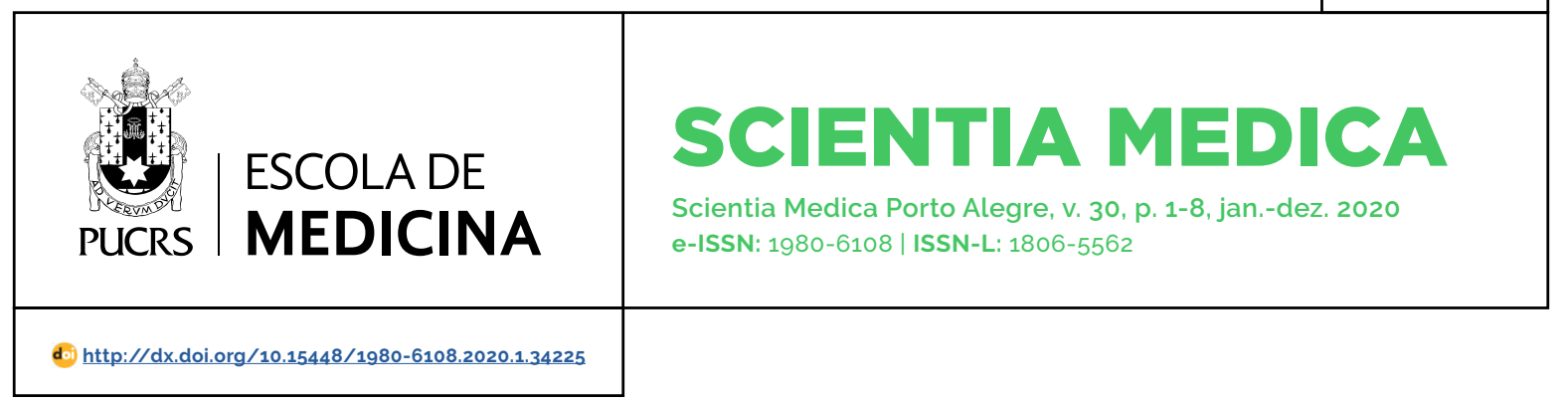

ARTIGO ORIGINAL

\title{
Leishmaniose visceral humana: um retrato da situação de casos em idosos e longevos
}

\author{
Human visceral leishmaniosis: a closure of the situation of cases in elderly and long- \\ lived people
}

\section{Ibrahim Clós Mahmud ${ }^{1}$ \\ orcid.org/0000-0002-2631-2964} ibrahim_mahmud@hotmail.com

\section{Carla Viero Kowalski ${ }^{1}$ \\ orcid.org/0000-0001-8394-7336 carlakowalski25@gmail.com}

\section{Paulo Renato Petersen Behar $^{2}$ \\ orcid.org/0000-0001-8382-6681 behar@ufcspa.edu.br}

\section{Claus Dieter Stobäus} orcid.org/0000-0002-5426-7742 claus.stobaus@adpppucrs.com.br

Recebido em: 21 mai. 2019. Aprovado em: 31 out. 2019 . Publicado em: 25 mai. 2020

\section{(c) (i)}

Artigo está licenciado sob forma de uma licença Creative Commons Atribuição 4.0 Internacional.

\section{Resumo}

Objetivo: analisar a atual situação epidemiológica brasileira e gaúcha da leishmaniose visceral (LVH), em idosos e verificar o coeficiente de letalidade.

Métodos: trata-se um estudo transversal realizado com fonte de dados secundária, em consulta à base de dados do Sistema de Informação de Agravos de Notificação (SINAN), de janeiro de 2013 a dezembro de 2017, de casos confirmados de leishmaniose visceral no Brasil e no Rio Grande do Sul, que foram tabulados em Excel e analisados com Epiinfo 7.0.

Resultados: casos de LVH em idosos têm aumentado nos últimos 5 anos, dentro do cenário brasileiro e gaúcho. Embora pouco divulgado, é frequente o comprometimento e o aumento da proporção de idosos que vão a óbito pelo LHV, que foi de $20,3 \%$ dos casos no periodo de 2013 a 2017, demonstrando a gravidade da infecção nesse público. Em nosso estudo também encontramos forte relação da idade com o aumento do coeficiente de letalidade, chegando a 46,87\% em 2016.

Discussão: uma das estratégias recentes no combate à LVH é o abate de cães que contêm o parasita responsável pela transmissão da doença, porém esse método de controle não tem sido muito efetivo. Com isso, constata-se que a medida imunoprofilática, através da vacina Leish-Tec ${ }^{\circledR}$, tem um efeito favorável no combate à doença somente em animais que não estão em áreas de alta transmissão.

Palavras-chave: leishmaniose visceral, idoso, idoso de 80 anos ou mais, infectologia, geriatria

\section{Abstract}

Aims: to analyze the current Brazilian and of the state of Rio Grande do Sul epidemiological situation of visceral leishmaniasis (LVH) in the elderly and verify the mortality coefficient.

Methods: This is a cross-sectional study with a secondary data source, taken from SINAN data from January 2013 to December 2017, with confirmed visceral leishmaniasis in Brazil and Rio Grande do Sul, which are tabulated in Excel and analyzed with Epiinfo 7.

Results: cases of LVH in the elderly have increased in the last 5 years, within the Brazilian and the state of Rio Grande do Sul settings. Although not widely reported, it is frequent to compromise and increase the proportion of elderly people who die from LVH, which was $20,3 \%$ of the cases in the period from 2013 to 2017 , demonstrating the seriousness of the infection in this public. In our study we also found a strong relation between age and the increase in the lethality coefficient, reaching $46.87 \%$ in 2016.

Discussion: One of the recent strategies in the fight against HVL is the slaughter of dogs that contains the parasite responsible for the transmission of the disease, but this method of control has not been very effective. Thus, the immunoprophylactic measurement by Leish-Tec ${ }^{\circledR}$ vaccine has a favorable effect in the fight 
against the disease only in animals that are not in high transmission areas.

Keywords: leishmaniasis, visceral, aged, aged 80 and over, infectious disease medicine, geriatrics.

ABREVIATURA: DIP, Doenças infecto-parasitárias; LVH, Leishmaniose Visceral Humana; SINAN, Sistema de Informação de Agravos de Notificação;

\section{Introdução}

O crescimento acelerado da população de idosos e do incremento da expectativa de vida em várias partes do mundo gera, como consequência, o aumento da prevalência de doenças, entre as quais se destacam as doenças negligenciadas. São doenças que não só prevalecem mais em condições de pobreza, mas também contribuem para a manutenção do quadro de desigualdade, já que podem representar uma forte entrave ao desenvolvimento dos paises. Como exemplos de doenças negligenciadas, podemos citar: dengue, doença de Chagas, esquistossomose, hanseniase, leishmaniose, malária, tuberculose, entre outras.

Segundo dados da Organização Mundial de Saúde, mais de um bilhão de pessoas estão infectadas com uma ou mais doenças negligenciadas, o que representa cerca de um sexto da população mundial cuja dimensão tem sido despercebida pela sociedade brasileira e cujo impacto não tem sido discutido adequadamente nos meios acadêmicos, menos ainda no âmbito das políticas de saúde (1).

Dentro desse contexto, faz-se necessário pensar na mudança de perfil epidemiológico e da transição demográfica que traz consigo uma transformação na carga de morbimortalidade entre idosos.

Seguindo o envelhecimento populacional global a transição demográfica carrega consigo uma transição na carga de morbimortalidade nessa faixa etária. A partir do modelo proposto por Abdel Omran há 30 anos, esse processo se caracteriza por basicamente três aspectos: a substituição das doenças infecto-parasitárias (DIP) pelas doenças crônicas não transmissiveis e causas externas, o deslocamentos da carga de morbimortalidade (doenças e óbitos) da população adulta para a idosa e a transformação para um cenário em que a morbidade predomine quando comparada com a mortalidade (2).

Em paises desenvolvidos esse processo ocorre como determinado e, em alguns paises latinoamericanos como o Chile, Cuba e Costa Rica, o mesmo também já foi experimentado (3). Logo, ao analisarmos a situação brasileira, os gráficos de transição não demonstram o esperado, assim como ocorre no México, segundo Chaimowicz, alguns aspectos principais os diferenciam de outros processos que ocorrem no mundo, sendo eles:

1. A não substituição das DIP pelas doenças crônicas não transmissíveis, mas sim, uma superposição delas, nas quais há um predomínio de ambas associadas às causas externas;

2. No Brasil ocorreu uma drástica redução das doenças imunopreveniveis (doenças infecciosas que já possuem vacinas especificas para sua prevenção). Reintrodução de outras DIP, como a dengue e a cólera, persistência das hepatites $\mathrm{B}$ e $\mathrm{C}$ e da esquistossomose e, ainda, um aumento da sindrome da imunodeficiência adquirida, indicando ainda uma contra transição epidemiológica no Brasil;

3. Finalmente, o processo não apresenta uma projeção de resolução rápida, mas ao contrário, demonstrando-se ser uma transição prolongada (3).

Pesquisadores vêm realizando trabalhos onde analisam a incidência de cada DIP e projetam sobre como será a incidência e a prevalência das mesmas nos próximos anos na população idosa $(4,5,6)$. Através disso, evidenciou-se que algumas delas entraram em declínio (malária e febre tifoide), outras terão tendência de se manter com as mesmas taxas como a tuberculose, hanseniase, leptospirose, meningite, tétano e esquistossomose. Entretanto estima-se que há uma tendência de aumento para 2020, das taxas de incidência de sindrome da imunodeficiência adquirida, leishmaniose visceral humana (LVH) e tegumentar, dengue, hepatites e doença de Chagas no Brasil. Porém as DIP de maior mortalidade listadas foram: diarreia e gastroenterite, sindrome da imunodeficiência adquirida, doença de Chagas, 
tuberculose e leishmaniose $(4,5,6)$.

No Brasil, estão presentes 14 das 17 doenças tropicais negligenciadas listadas pela Organização Mundial da Saúde. A LVH é uma delas. Também conhecida como calazar, tem como agente etiológico parasitas do complexo Leishmania donovani. O quadro clínico caracteriza-se basicamente por febre de longa duração, perda de peso, astenia, anemia (chegando a pancitopenia), hepatoesplenomegalia, dentre outros sintomas. Com base no exposto, o seguinte trabalho visa analisar a atual situação epidemiológica brasileira e gaúcha da LVH em idosos e verificar o coeficiente de letalidade da mesma $(7,8)$.

\section{Método}

A LVHé uma doença de notificação compulsória em todo o território nacional, conforme Lei $n{ }^{\circ}$ 6.259 de 30/10/1975 e Portaria GM/MS 104 de 25/01/2011 (9, 10). O Ministério da Saúde possui um banco de dados na internet, o Sistema de Informação de Agravos de Notificação (SINAN) (11). Esse sistema é capaz de dividir os casos notificados conforme o desfecho nos seguintes campos: cura (cura da enfermidade confirmada pelo médico responsável), abandono (abandono do tratamento da enfermidade), transferência (paciente mudou-se de município/estado ou região), óbito e ignorado/campo em branco.

Trata-se de um estudo transversal realizado com fonte de dados secundária, SINAN, em que foram coletados entre os meses de janeiro e março de 2019, os dados de janeiro de 2013 a dezembro de 2017, dos casos confirmados de leishmaniose visceral no Brasil e no Rio Grande do Sul, separados pela faixa etária, evolução e municipio de notificação (11). Dessa forma, foram obtidos 30 arquivos, contendo dados dos casos com as variáveis descritas acima, por ano. Esses dados foram tabulados em Excel e analisados através do Epiinfo 7.0. Realizou-se a análise do coeficiente de letalidade, sendo ela realizada a partir da seguinte fórmula: $n .^{\circ}$ de óbitos devido à doença/n. ${ }^{\circ}$ de casos confirmados com a doença $x$ 100. Por se tratar de uma análise secundária de dados publicamente disponiveis, sem dados pessoais, o projeto não necessitou ser submetido à Comissão de Ética e Pesquisa.

\section{Resultados}

Os casos de LVH em idosos têm apresentado crescimento nos últimos 5 anos, dentro do cenário brasileiro. Na Tabela 1 evidencia-se esse aumento, já que em 2013 foram notificados 249 casos e, em 2017, esse número passou para 390. A faixa etária que esse número mais cresceu foi a de longevos, que passou de 34 casos em 2013 para 71 em 2017. Ainda, do total de casos confirmados de LVH no Brasil de 2013 a 2017, 21,8\% deles foram em idosos.

TABELA 1 - Casos confirmados de Leishmaniose Visceral Humana Notificados no Brasil entre 2013 e 2017 no SINAN, distribuidos a partir de 60 anos de idades

\begin{tabular}{ccccccc}
\hline Faixa etária & $\mathbf{2 0 1 3}$ & $\mathbf{2 0 1 4}$ & $\mathbf{2 0 1 5}$ & $\mathbf{2 0 1 6}$ & $\mathbf{2 0 1 7}$ & Total \\
\hline $\mathbf{6 0 - 6 9}$ & 136 & 167 & 153 & 194 & 212 & 862 \\
$\mathbf{7 0 - 7 9}$ & 79 & 95 & 94 & 92 & 107 & 467 \\
$\mathbf{8 0} \mathbf{e}+$ & 34 & 38 & 34 & 32 & 71 & 209 \\
Total & 249 & 300 & 281 & 318 & 390 & 1.538 \\
$\%^{*}$ & 7,1 & 8 & 7,9 & 9,2 & 8,6 & 21,8 \\
\hline
\end{tabular}

*Total de casos em idosos/ Total de casos incluindo todas as faixas etárias. SINAN: Sistema de Informação de Agravos de Notificação.

Fonte: construida pelos autores através de dados do SINANWEB - 2018. 
Na Tabela 2 apresentam-se os casos acumulados dos últimos cinco anos, distribuidos conforme a evolução ou desfecho do agravo. Verifica-se que 54,6\% dos casos alcançaram a cura da doença, porém 20,3\% foram a óbito por LVH e, ainda, 7,1\% foram a óbito por outras causas, estando presente as morbidades do paciente idoso. Os dados demonstram também que quanto mais velho for o paciente, maior será a chance de óbito por LVH e outras causas, e menor será a chance de cura. Nessa situação os longevos representaram $30,7 \%, 10,1 \%$ e $45,4 \%$ sobre o total de casos, respectivamente.

TABELA 2 - Casos confirmados de Leishmaniose Visceral Humana Notificados no Brasil entre 2013 e 2017 no SINAN, distribuidos a partir de 60 anos de idades e segundo sua evolução

\begin{tabular}{|c|c|c|c|c|c|c|c|}
\hline $\begin{array}{l}\text { Faixa } \\
\text { etária }\end{array}$ & $\begin{array}{l}\text { Ignora/ } \\
\text { Branco }\end{array}$ & Cura & Abandono & $\begin{array}{l}\text { Óbito } \\
\text { por LVH }\end{array}$ & $\begin{array}{l}\text { Óbito } \\
\text { por outra } \\
\text { causa }\end{array}$ & Transferência & Total \\
\hline $60-69, n(\%)$ & $125(14,5)$ & $503(58,4)$ & $6(0,7)$ & $136(15,8)$ & $52(6,0)$ & $40(4,6)$ & $862(56)$ \\
\hline 70-79, n(\%) & $55(11,8)$ & $242(51,8)$ & $2(0,4)$ & $113(24,2)$ & $35(7,5)$ & $20(4,3)$ & $467(30,4)$ \\
\hline$\geq 80, \mathrm{n}(\%)$ & $17(8,2)$ & $95(45,4)$ & $2(0,9)$ & $64(30,7)$ & $21(10,1)$ & $10(4,7)$ & $209(13,6)$ \\
\hline Total n(\%) & $197(12,8)$ & $840(54,6)$ & $10(0,7)$ & $313(20,3)$ & $108(7,1)$ & $70(4,5)$ & 1.538 \\
\hline$\%^{*}$ & 6,7 & 6,6 & 7,2 & 23 & 24,1 & 5,6 & 21,8 \\
\hline
\end{tabular}

*Total de casos em idosos/ Total de casos incluindo todas as faixas etárias. SINAN: Sistema de Informação de Agravos de Notificação.

Fonte: construida pelos autores através de dados do SINANWEB - 2018.

Na Tabela 3 evidencia-se que o coeficiente de letalidade da LVH no Brasil em pacientes idosos é diretamente relacionado à idade do indivíduo, na qual em longevos os valores são maiores chegando em 46,87\% em 2016. De 2013 a 2017 o coeficiente foi de $15,77 \%$ em idosos de 60 a 69 anos, 24,19\% naqueles de 70 a 79 anos e 30,62\% em longevos.

TABELA 3 - Coeficiente de letalidade* da Leishmaniose Visceral Humana em idosos no Brasil no período de 2013 a 2017 (dados expressos em percentuais).

\begin{tabular}{ccccccc}
\hline Faixa etária & $\mathbf{2 0 1 3}$ & $\mathbf{2 0 1 4}$ & $\mathbf{2 0 1 5}$ & $\mathbf{2 0 1 6}$ & $\mathbf{2 0 1 7}$ & $\mathbf{2 0 1 3} \mathbf{2 0 1 7}$ \\
\hline $\mathbf{6 0 - 6 9}$ & 16,17 & 14,97 & 18,18 & 19,27 & 13,48 & 15,77 \\
$\mathbf{7 0 - 7 9}$ & 20,25 & 22,10 & 26,59 & 26,08 & 27,35 & 24,19 \\
$\mathbf{8 0} \mathbf{e}$ & 26,47 & 34,21 & 20,58 & 46,87 & 30,13 & 30,62 \\
Total & 18,87 & 19,66 & 21,40 & 23,89 & 20,30 & 20,35 \\
\hline
\end{tabular}

*Total de óbitos por leischmaniose visceral humana em idosos/ Total de casos de leischmaniose visceral humana em idosos. Fonte: construída pelos autores através de dados do SINANWEB - 2018. 
Quando analisados os dados separando-os por regiões, verifica-se que $46,5 \%$ dos casos em idosos são provenientes da região Nordeste do Brasil, enquanto a região Sul apresenta somente $0,8 \%$ do total dos casos. Entretanto, a representação da doença nessa faixa etária difere muito entre elas, onde no Nordeste a LVH nos idosos representa somente $6,7 \%$ dos casos enquanto na região Sul eles representam 24,5\% (Tabela 4).

TABELA 4 - Casos confirmados de Leishmaniose Visceral Humana Notificados no Brasil entre 2013 e 2017 no SINAN, distribuidos a partir de 60 anos de idade e conforme região de notificação

\begin{tabular}{ccccccc}
\hline $\begin{array}{c}\text { Faixa } \\
\text { Etária }\end{array}$ & $\begin{array}{c}\text { Região } \\
\text { Norte }\end{array}$ & $\begin{array}{c}\text { Região } \\
\text { Nordeste }\end{array}$ & $\begin{array}{c}\text { Região } \\
\text { Sudeste }\end{array}$ & $\begin{array}{c}\text { Região } \\
\text { Sul }\end{array}$ & $\begin{array}{c}\text { Região } \\
\text { Centro- } \\
\text { Oeste }\end{array}$ & Total \\
\hline $\mathbf{6 0 - 6 9 , \mathbf { n } ( \% )}$ & $82(9,5)$ & $419(48,6)$ & $252(29,2)$ & $6(0,7)$ & $103(12)$ & $862(56)$ \\
$\mathbf{7 0 - 7 9 , \mathbf { n } ( \% )}$ & $47(10)$ & $213(45,7)$ & $145(31)$ & $6(1,3)$ & $56(12)$ & $467(30,4)$ \\
$\mathbf{2 8 0 , \mathbf { n } ( \% )}$ & $16(7,7)$ & $82(39,2)$ & $79(37,8)$ & $1(0,5)$ & $31(14,8)$ & $209(13,6)$ \\
Total, $\mathbf{n}(\%)$ & $145(9,4)$ & $714(46,5)$ & $476(31)$ & $13(0,8)$ & $190(12,3)$ & 1.538 \\
\%* & 4,8 & 6,7 & 13 & 24,5 & 13,6 & 8,2 \\
\hline
\end{tabular}

* (Total de casos em idosos/ Total de casos incluindo todas as faixas etárias). SINAN: Sistema de Informação de Agravos de Notificação

Fonte: construida pelos autores através de dados do SINANWEB - 2018

No Rio Grande do Sul, a carga de LVHé muito menor. Nos últimos 5 anos, foram notificados somente 18 casos e 9 (50\%) deles foram em idosos (6o-69 anos: 5 casos; 70-79: 3 e apenas 1 caso em longevos). Isso se explica pela recente introdução da doença no Estado. Quanto à evolução da doença, houve somente 1 óbito (representando $25 \%$ do total de óbitos por LVH) e 7 casos que alcançaram a cura da doença. Cabe ressaltar que do total de casos de LVH em idosos, 33\% (3) deles foram registrados em Porto Alegre, capital do RS, 33\% (3) em São Borja, 22\% (2) em Uruguaiana e $11 \%$ (1) em Santa Maria, demonstrando que mais da metade dos casos (55.5\%) foram notificados em região de fronteira com a Argentina.

\section{Discussão}

A LVHé uma doença negligenciada, de carácter endêmico no Brasil, transmitida pelo vetor Lutzomya longipalpis, conhecido popularmente como mosquito palha ou birigui. Diversos estudos demonstram a epidemiologia dos casos em adultos e crianças, sendo inclusive muito investigado o seu quadro clínico. Porém, encontram-se pouco trabalhos que tratam especificamente do paciente idoso portador de LVH ou estudos que relatem o comportamento da mesma em longevos, talvez seja explicado pela "ideia antiga" da transição epidemiológica clássica (7).

Outro fator associado a essa questão está na relação do estudo da leishmaniose humana com a canina, a qual não pode ser realizado de forma separada. O cão, por ser de grande importância como animal de estimação, passa a ser um enorme reservatório. O diagnóstico da leishmaniose visceral foi beneficiado recentemente pelo aparecimento de teste de realização prática e rápida denominado teste imunocromatográfico rk3 (12).

Mesmo assim, observa-se, na Tabela 3, um aumento do coeficiente de letalidade. Um aspecto limitante em relação ao diagnóstico da leishmaniose no ser humano se deve ao fato dos estudos estarem mais concentrados na patologia 
e na imunorregulação da doença, havendo poucos instrumentos na detecção precoce da doença (13).

Nessa direção, corrobora-se com os autores ao mencionarem que as questões socioambientais interferem no processo de adoecimento. Isso fica evidente ao analisar que os casos de LVH em idosos vêm aumentando nos últimos anos, chegando a exatos 1.538 casos nos últimas cinco anos, representando $21,8 \%$ do total de casos no Brasil. Muito se deve à falta de Educação em Saúde/Educação para a Saúde dessa população, pois atualmente as políticas de saúde não estão direcionadas a essa faixa etária, já destacavam Mosquera e Stobäus (14).

As leishmanioses são consideradas um problema de saúde pública, devido às suas magnitudes, incapacidades e complexidades clinicas. Afetam, predominantemente, os mais pobres, sobretudo em paises em desenvolvimento. Essa patologia requer um esforço coletivo e multiprofissional entre governos, organizações, instituições e sociedade para seu controle.

Em 2016, os paises Membros da Organização Pan-Americana da Saúde- Organização Mundial de Saúde aprovaram o Plano de Ação para Eliminação das Doenças Negligenciadas e Medidas Posteriores à Eliminação 2016-2022 (15). Fato esse que demostra a preocupação em relação à crescente incidência de leishamaniose.

Embora pouco divulgado, é frequente o comprometimento e o aumento da proporção de idosos que vão a óbito pelo LHV. No periodo de 2013 a 2017, eles contabilizaram 20,3\% dos casos e, ainda, 7,1\% foram a óbito por outras causas, demonstrando a gravidade da infecção nesse público. Logo, questiona-se sobre o conhecimento do comportamento da doença nessa população, que por questões imunológicas e pela carga de morbidades presentes os torna diferentes na abordagem, inclusive com uma maior porcentagem de interações medicamentosas (16).

Vale e Cavalcante demonstraram que a cura tende a estar diminuida e a letalidade aumentada com a idade. Logo, pacientes com 60 anos ou mais têm uma chance maior de falecer devido à doença ou a alguma morbidade que apresente
(17). Em nosso estudo também encontramos forte relação da idade com o aumento do coeficiente de letalidade, deixando os longevos com o maior coeficiente de letalidade, chegando a 46,87\% em 2016 e 30,62\% na somatória dos últimos 5 anos. Em geral, a leishmaniose visceral acarreta a perda de mais anos de vida por conta de morte prematura, justamente pela alta letalidade da doença: 7,4\% dos casos diagnosticados levam ao óbito (18).

Botelho e Natal trazem em seu discurso as hipóteses da maior mortalidade em idosos e, ainda, questionam a falta de pesquisas na área, onde em seus dados os idosos contribuíram com apenas $9 \%$ dos casos e, os óbitos entre eles, alcançaram 39\% do total de mortes pela doença (19). Acredita-se que as altas taxas de mortalidade possam estar relacionadas à diminuição da capacidade de resposta do sistema imunológico e, ao conhecimento dos profissionais de saúde, que associam a LVH a um agravo predominantemente infantil, diminuindo as suspeitas em idosos (19).

Quando comparadas as distribuições da LVH em idosos nas regiões brasileiras, dois fatores são importantes de ser analisados. Primeiramente, a maior incidência de casos na região Nordeste, que por fatores sociais, culturais e econômicos, possui esse alto indice. Porém, a região Sul apresenta a maior representação de idosos doentes, com $24.5 \%$ das notificações, algo que pode ser explicado pelo maior número de idosos na região Sul e pela maior esperança de vida nestes estados, principalmente no Rio Grande do Sul e Santa Catarina (20).

Ao analisarmos a distribuição dos casos em idosos no Rio Grande do Sul, verifica-se que $55.5 \%$ deles são em região de fronteira e 33.3\% na capital, demonstrando que o ingresso da doença vem pela região de fronteira da Argentina com o Brasil. Os municipios de São Borja, Uruguaiana e Itaqui, no Rio Grande do Sul, apresentaram casos autóctones em 2008 e 2009. Acredita-se que essa situação se expandiu em razão do trânsito de pessoas com seus mascotes vindos da Argentina, pois registraram-se casos de LVH em Posadas, na Argentina $(21,22)$. 
Uma das estratégias recentes no combate à leishmaniose visceralé o abate de cães que contêm o parasita responsável pela doença, porém esse método de controle não tem sido efetivo. Com isso, constata-se que a medida imunoprofilática através da vacina Leish-Tec ${ }^{\circledR}$ tem um efeito favorável no combate à doença somente em animais que não estão em áreas de alta transmissão (13).

Pesquisas realizadas em São Tomé (fronteira da Argentina com o Brasil) e São Borja constataram, até o ano de 2010, cinco casos de LVH (8). Em Porto Alegre, Rio Grande do Sul, o primeiro caso autóctone foi registrado em setembro de 2016 , levando equipes de saúde municipais a capacitar profissionais para o diagnóstico precoce (23).

Evidenciou-se que o modelo de saúde vigente ainda está fortemente centrado na doença e sua medicalização, apesar das medidas de reorientação que vêm se intensificando com a consolidação em se trabalhar a prevenção de agravos. As doenças negligenciadas requerem envolvimento indistinto de toda a sociedade para que se minimizem os danos e se consiga uma gradativa redução da mortalidade gerada por elas.

Os recursos disponiveis em nosso país são escassos e transformá-los em resultados compativeis com as necessidades sociais é um desafio permanente. Trata-se de um desafio labirintico a exigir competência e criatividade, para diagnosticá-lo em todos os seus meandros e formular a receita para superá-lo.

Com ampla distribuição geográfica as leishmanioses são doenças de elevada incidência em todo o País e constituem um problema de saúde pública, pois requerem um grande esforço político, técnico e operacional para manter o desenvolvimento sistemático de ações de vigilância em saúde para o controle dessas doenças. Outro fator preponderante observado no estudo foi a lacuna de publicações voltadas aos idosos, parcela da população emergente, e muitas vezes, negligenciada.

A LVH é um doença grave, por vezes, negligenciada, e que requer atenção por parte das autoridades de saúde pública. No RS, a introdução da doença é algo ainda novo, porém encontra-se em franco crescimento. A população idosa está sendo cada vez mais afetada pela mesma, visto que os casos seguem aumentando e o coeficiente de letalidade também.

\section{Referências}

1. World Health Organization. Integrating neglected tropical diseases into global health and development: fourth WHO report on neglected tropical diseases. Geneva: WHO; 2017.

2. Onram AR. The Epidemiologic Transition: A Theory of the Epidemiology of Population. Change Milbank Q. 2005:83(4):731-57. https://doi.org/10.1111/j. 1468-0009.2005.00398.x

3. Chaimowicz F. Saúde do idoso. 2. ed. Belo Horizonte: NESCON UFMG; 2013

4. Luna EJA, Silva JJB. Doenças transmissiveis, endemias, epidemias e pandemias. In: Fundação Oswaldo Cruz. A saúde no Brasil em 2030 - prospecção estratégica do sistema de saúde brasileiro: população e perfil sanitário. Rio de Janeiro: Fiocruz/Ipea/Ministério da Saúde/Secretaria de Assuntos Estratégicos da Presidência da República, 2013;2:123-76. https://doi. org/10.1590/s1413-81232014000100032

5. Araújo PR. Evolução da mortalidade por doenças infecciosas e parasitárias [dissertation]. [Rio de Janeiro]: Escola Nacional de Saúde Pública Sergio Arouca; 2015. 62 p. https://doi.org/10.5327/ Z1679443520180267

6. Ikuta IM. Aspectos epidemiológicos das doenças infecciosas em idosos no estado do Pará [thesis]. [Belém]: Universidade Federal do Pará; 2017. 132 p.

7. Brasil. Ministério da Saúde. Leishmaniose visceral: recomendações clínicas para redução da letalidade [Internet]. Brasília; 2011 [updated 2011; cited 2018 Jul 10]. Available from: http://sbmt.org.br/portal/wp-content/ uploads/2013/01/lv_reducao_letalidade_web.pdf.

8. Mahmud IC, Valese AM, Pedrollo ML, Malvera R, Irrazábal S, Galarza A. Leishmaniasis Visceral Infantil: Reporte de Caso y Revisión de Literatura1. Rev. Contexto \& Saúde 2014; 13(24-25):27-36

9. Brasil. Lei n. ${ }^{\circ}$ 6.259, de 30 de outubro de 1975. Dispõe sobre a organização das ações de vigilância epidemiológica, sobre o Programa Nacional de Imunização e estabelece normas relativas à notificação compulsória de doenças e dá outras providências. Diário Oficial da União [internet] 1975 out 31 lupdated 1975; cited 2019 march];209. Seção 1, (Pt 1): 014433 1. Available from: http://WwW. planalto.gov.br/ccivil_03/leis/L6259.htm https://doi. org/10.1590/0034-716719750003000001 
10. Brasil. Ministério da Saúde. Portaria n. 104, de 25 de janeiro de 2011. Define as terminologias adotadas em legislação Nacional, conforme o disposto no Regulamento Sanitário Internacional 2005 (RSI 2005), a relação de doenças, agravos e eventos em saúde pública de notificação compulsória em todo o território nacional e estabelece fluxo, critérios, responsabilidades e atribuições aos profissionais e serviços de saúde. Diário Oficial da União [internet]. 2011 Jan 26 [updated 2011; cited 2019 March]; Available from: http://bvsms.saude.gov.br/bvs/saudelegis/gm/2011/prt0104_25_01_2011.html https://doi. org/10.1590/s0102-311×2011000400008

11. SINANWEB. Dados epidemiológicos [internet]. Brasilia; 2018 [updated 2018 Nov; cited 2018 Nov]. Available from: http://Www2.datasus.gov.br/DATA-

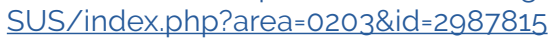

12. Boelaert M, Verdonck K, Menten J, Sunyoto T, van Griensven J, Chappuis F, Rijal S. Rapid tests for the diagnosis of visceral leishmaniasis in patients with suspected disease. Cochrane Database of Systematic Reviews 2014;20(6):1-119. https://doi. org/10.1002/14651858.cdoog135.pub2

13. Grimaldi G Jr, Teva A, dos-Santos CB, Santos FN, Pinto Id-S, Fux B, et al. (2017) Field trial of efficacy of the Leish-tec $(\mathbb{R}$ vaccine against canine leishmaniasis caused by Leishmania infantum in an endemic area with high transmission rates. PLoS ONE 12(g):e0185438. https://doi.org/10.1371/journal. pone. 0185438

14. Mosquera JMM, Stobäus CD. Educação para a Saúde. 2. ed. Porto Alegre: D. C. Luzzatto; 1984.

15. Pan-American Health Organization. Informe Epidemiológico das Américas Informe de Leishmanioses N 5 - Abril, 2017. [internet]. OPAS; 2017 [updated 2017 Nov; cited 2018 Nov]. Available from: http://iris.paho.org/xmlui/bitstream/handle/123456789/34113/informe_leishmanioses_5_por. pdf? sequence $=1 \&$ is Allowed $=y$

16. Freitas EV, Py L. Tratado de geriatria e gerontologia. 4. ed. Rio de Janeiro: Guanabara Koogan; 2018.

17. Cavalcante IJM, Vale MR. Aspectos epidemiológicos da leishmaniose visceral (calazar) no Ceará no período de 2007 a 2011. Rev. bras. epidemiol. 2014:17(4):911-24. https://doi.org/10.1590/18094503201400040010

18. Brasil. Ministério da Saúde. Departamento de Ciência e Tecnologia. Secretaria de Ciência, Tecnologia e Insumos Estratégicos. Doenças negligenciadas: estratégias do Ministério da Saúde. Rev.Saúde Publica. 2010;44(1):200-02. https://doi.org/10.1590/ s0034-89102010000100023

19. Botelho ACA, Natal D. First epidemiological description of visceral leishmaniasis in Campo Grande, State of Mato Grosso do Sul. Rev. Soc. Bras. Med. Trop. 2009; 42(5): 503-8. https://doi.org/10.1590/ s0037-86822009000500006
20. Instituo Brasileiro de Geografia e Estatística (IBGE). Estimativas populacionais. Brasilia: IBGE, 2018 [internet]. Brasilia; 2018 [update 2018 Nov; cited 2019 Jan]. Available from: https://www.ibge.gov.br/apps/ populacao/projecao/

21. Salomón OD, Fernández MS, Santini MS, Saavedra S, Montiel N, Ramos MA, et al. Distribución de Lutzomya longipalpis em la mesopotamia argentina, 2010. Rev Med. 2011;71(1): 22-6.

22. Salomón OD, Ramos LK, Quintana MG, Acardi SA, Santini MS, Schneider A. Distribución de vectores de leishmaniasis visceral en la provincia de Corrientes. 2008. Rev Med. 2009:69(6):625-30.

23. Porto Alegre. Secretaria Municipal de Saúde. Relatório de Gestão. $1^{\circ}$ quadrimestre 2017 [internet]. Porto Alegre; 2017 [updated 2017 Dez; cited 2018 jul]. Available from: http://lproweb.procempa.com.br/

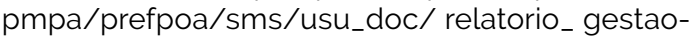
1quadrimestre_2017.pdf https://doi.org/10.15689/ ap.2017.1602.03

\section{Endereço para correspondência}

\section{Ibrahim Clós Mahmud}

Pontificia Universidade Católica do Rio Grande do Sul Av. Ipiranga, $6.681,7^{\circ}$ andar, sala 703

Jardim Botânico, 90619-900

Porto Alegre, RS, Brasil 